\title{
VISUAL SIGNS AND SYMPTOMS OF MULTIPLE SYSTEM ATROPHY (MSA)
}

\author{
R.A. Armstrong D.Phil.
}

Vision Sciences, Aston University, Birmingham, B4 7ET, UK

(Tel: 0121-204-4102; Fax: 0121-204-4048; Email: R.A.Armstrong@aston.ac.uk) 


\section{$\underline{\text { List of abbreviations }}$}

$\begin{array}{ll}\text { APO E } & \text { Apolipoprotein E } \\ \text { CBD } & \text { Corticobasal degeneration } \\ \text { DLB } & \text { Dementia with Lewy bodies } \\ \text { ERG } & \text { Electroretinogram } \\ \text { GABA } & \gamma \text {-amino-butyric acid } \\ \text { GCI } & \text { Glial cytoplasmic inclusions } \\ \text { iREM } & \text { Idiopathic rapid eye movement disorder } \\ \text { MSA } & \text { Multiple system atrophy } \\ \text { MSA-C } & \text { Multiple system atrophy (cerebellar subtype) } \\ \text { MSA-P } & \text { Multiple system atrophy (parkinsonian subtype) } \\ \text { PD } & \text { Parkinson's disease } \\ \text { PSP } & \text { Progressive supranuclear palsy } \\ \text { VEP } & \text { Visual evoked potential } \\ \text { VOR } & \text { Vestibulo-ocular reflex }\end{array}$




\begin{abstract}
Multiple system atrophy (MSA) is a rare movement disorder and a member of the 'parkinsonian syndromes’ which also includes Parkinson's disease (PD), progressive supranuclear palsy (PSP), dementia with Lewy bodies (DLB), and corticobasal degeneration (CBD). MSA is a complex syndrome in which patients exhibit a variety of signs and symptoms including parkinsonism, ataxia, and autonomic dysfunction. It can be difficult to separate MSA from the other parkinsonian syndromes but if ocular signs and symptoms are present they may aid differential diagnosis. Typical ocular features of MSA include blepharospasm, excessive square-wave jerks, mild to moderate hypometria of saccades, impaired vestibular-ocular reflex (VOR), nystagmus, and impaired event-related evoked potentials. Less typical features include slowing of saccadic eye movements, the presence of vertical gaze palsy, visual hallucinations, and an impaired electroretinogram (ERG). Aspects of primary vision such as visual acuity, colour vision, or visual fields are usually unaffected. Management of the disease to deal with problems of walking, movement, daily tasks, and speech problems is important in MSA. Optometrists can work in collaboration with the patient and health care providers to identify and manage the visual deficits of the patient. A more specific role for the optometrist is to correct vision to prevent falls and to monitor the anterior eye to prevent dry eye and control blepharospasm.
\end{abstract}

Key words: Multiple system atrophy (MSA), parkinsonism, autonomic dysfunction, dementia with Lewy bodies (DLB), progressive supranuclear palsy (PSP) 


\section{Introduction}

Multiple system atrophy (MSA) is a rare movement disorder and a member of a group of neurodegenerative diseases known as the 'parkinsonian syndromes'. Characteristic of these syndromes is that patients exhibit the symptoms of 'parkinsonism', viz. a range of problems involving movement, most typically manifest in Parkinson's disease (PD) itself ${ }^{1}$, but also seen in progressive supranuclear palsy (PSP) ${ }^{2}$, and to some extent in dementia with Lewy bodies (DLB) ${ }^{3}$, and corticobasal degeneration (CBD). ${ }^{4}$

MSA is derived from three previously described disease entities, viz. olivopontocerebellar atrophy, striato-nigral degeneration, and Shy-Drager syndrome. ${ }^{5}$ These three diseases were originally regarded as distinct but are now considered to be subtypes of MSA. ${ }^{6}$ The classical symptoms of MSA include parkinsonism, ataxia, and autonomic dysfunction. ${ }^{6}$ Ataxia describes a gross lack of coordination of muscle movements while autonomic dysfunction involves a variety of systems that regulate unconscious bodily functions such as heart rate, blood pressure, bladder function, and digestion. It can be difficult to separate MSA from the other parkinsonian syndromes but if ocular signs and symptoms are present, then they may aid differential diagnosis.

The main objectives of this review are: (1) to describe the general features of MSA, (2) to evaluate the visual signs and symptoms reported in the disorder, (3) to discuss those visual features which may help in differential diagnosis, and (4) to consider the role of the optometrist in managing the symptoms of the disease.

\section{General features}

\section{Incidence}

The average annual incidence of MSA is 3.0/100,000 of the population and the median survival time 8.5 years $^{7}$, an older age of onset being associated with a shorter survival time. The disorder is slightly more common in males than females (Male:Female ratio 1.3:1). Symptoms usually begin early in the fifth decade of life ${ }^{8}$ and mean age of onset is 54.2 years (range $31-78$ years). ${ }^{9}$ 
Signs and symptoms

A list of the more common general signs and symptoms of MSA is shown in Table 1. The most consistent clinical syndrome is parkinsonism (87\% of patients), followed by autonomic dysfunction (74\%), cerebellar ataxia (54\%), and pyramidal tract signs (49\%). ${ }^{8}$ The most common sign at presentation is an akinetic-rigid syndrome resembling that of $\mathrm{PD}^{1}$, and observed in $62 \%$ of cases, while cerebellar ataxia and genito-urinary dysfunction are found in $22 \%$ and $9 \%$ of cases respectively. Erectile dysfunction may be one of the first signs in males.

Of the autonomic system problems, urinary dysfunction is often more common and an earlier manifestation of the disease than orthostatic hypotension (postural low blood pressure). ${ }^{10}$ In addition to visual sensory symptoms, olfaction can be impaired in MSA, although deficits are usually less severe than in PD. ${ }^{8}$ Patients with either predominantly cerebellar signs or parkinsonism are regarded as distinct subtypes of MSA and designated as 'MSA-C' and 'MSA-P' respectively. Neuropsychiatric features, most usually depression, are present in about $40 \%$ of patients ${ }^{11}$ and such symptoms can also occur at presentation. ${ }^{12}$ There is a moderate intellectual impairment in some patients but severe loss of cognitive function is unusual and dementia is rare. ${ }^{8}$ MSA has a faster progression than PD and there is usually no remission from the disease. ${ }^{7}$

\section{Diagnosis}

The clinical diagnosis of MSA can be difficult owing to the overlap of clinical symptoms with other parkinsonian syndromes. 'Consensus criteria', i.e., those agreed by a panel of experts in the field have been established to diagnose MSA. ${ }^{13,14}$ Hence, the clinical symptoms of the disease are classified into four 'domains', viz., (1) autonomic and urinary dysfunction, (2) parkinsonism, (3) cerebellar ataxia, and (4) corticospinal dysfunction. A diagnosis of 'possible' MSA requires the presence of at least one of these criteria as a major feature plus at least two other features from separate domains. 'Probable' MSA requires the presence of autonomic failure, a poor response to the drug levodopa ( $L$-dopa), and either the presence of parkinsonism or 
cerebellar ataxia. A diagnosis of 'definite' MSA, however, requires pathological confirmation at post-mortem.

\section{Gross neuropathology}

The cerebral cortex in MSA is usually significantly smaller than in control patients of similar age, the degree of atrophy reflecting disease duration rather than patient age. ${ }^{15}$ There may be a specific and progressive cerebral atrophy affecting the frontal lobes ${ }^{16}$ and the motor/premotor areas. ${ }^{17}$ In the cerebellum, pathology is more evident in the vermis than the hemispheres. ${ }^{18}$ In the midbrain, the substantia nigra and locus caeruleus are often pale due to loss of pigment. In addition, there may be atrophy and discolouration of the striatum specifically affecting the putamen, and this pathology is especially evident in the MSA-P subtype (Fig 1).

\section{Cellular pathology}

The cellular neuropathology of MSA is characterised by neuronal loss, gliosis, and myelin pathology affecting the putamen, caudate nucleus, external pallidum, substantia nigra, locus caeruleus, inferior olive, pontine nuclei, Purkinje cells of the cerebellum, and intermediate cell columns of the spinal $\operatorname{cord}^{6,8}$ (Fig 2). The most characteristic pathological change is the presence of abnormal protein aggregates, termed glial cytoplasmic inclusions (GCI) found mainly in oligodendrocytes and first described in $1989 .{ }^{19}$ This pathology affects the substantia nigra, striatum, inferior olivary nucleus, pontine nuclei, and cerebellum ${ }^{20}$ (Fig 3). The GCI comprise abnormal aggregations of silver-positive, $10-15 \mathrm{~nm}$ diameter coated filaments, the major molecular constituent of which is the protein $\alpha$-synuclein. $\alpha$-Synuclein is also found in the Lewy bodies (LB) characteristic of $\mathrm{PD}^{1}$ and in $\mathrm{DLB}^{3}$ and unites these disorders with MSA as a major molecular group of neurodegenerative disease, viz. the 'synucleinopathies'.21

\section{Causes}

The cause of MSA has not been established. Nevertheless, the abundance of $\alpha$ synuclein-immunoreactive GCI in various brain areas suggests a fundamental defect 
of the cytoskeleton affecting glial cells. ${ }^{22}$ Pathological changes in human patients, however, do not appear to have a simple genetic basis. No nucleotide alterations have been found to date in the entire coding region of the $\alpha$-synuclein gene in MSA. It is possible, however, that there could be mutations in the regulatory or intronic regions of the gene which could contribute to the cause of the disease. ${ }^{23}$ Two further genes have been implicated in MSA, viz., ZNF 231, a novel brain-specific gene encoding a nuclear protein involved in transcription elevation ${ }^{24}$ and (Src homology 2 domain containing) transforming protein 2 located on chromosome 19 (19p13.3), a gene involved in signal transduction. ${ }^{25}$ Allelic variation of the apolipoprotein E (APO E) gene, which may be a risk factor in DLB, does not appear to be involved in MSA. ${ }^{26}$ Alternatively, it has been suggested that MSA may have an environmental cause, but there is little evidence to support this conclusion. ${ }^{27}$

\section{Treatment}

There are no treatments which will prevent the progression of MSA and treatment of the disorder often involves medication and life-style changes to help manage the symptoms. Three types of treatment are usually involved. First, corticosteroids such as fludrocortisone or minodrine are used to raise blood pressure. L-threodihydroxyphenylserine, which is well tolerated by patients, can also be used for this purpose. $^{28}$ Second, L-dopa and carbidopa are given to reduce the symptoms of parkinsonism, but not all MSA patients will be responsive. Third, treatments are used to manage difficulties in swallowing and breathing and to manage bladder care. Many of these treatments, especially those used to control parkinsonism, can have adverse ocular effects. ${ }^{1}$ Hence, patients treated with L-dopa and carbidopa may develop mydriasis followed by miosis, lid ptosis and blepharospasm. In the OPCA variant of MSA, there is a reduction in $\gamma$-amino-butyric acid (GABA) in the brain and cerebral spinal fluid. ${ }^{29}$ In two cases, treatment with gabapentin improved ataxia in one case while in the other, there was an improvement in severe cerebellar dysarthria and the patient also exhibited less oscillopsia and better vision.

\section{Visual signs and symptoms}


Several of the brain areas affected in MSA are involved in visual function including the cerebral cortex, striatum, midbrain, and cerebellum (Fig 4). A variety of visual problems have therefore been reported in MSA and the major signs and symptoms are summarised in Table 2.

\section{Primary vision}

There is little detailed information concerning changes in visual acuity (VA) in MSA. Patients with other forms of parkinsonism ${ }^{1-3}$, however, often complain of poor vision especially as the disease progresses, and this may also be present in MSA. In PD, vision has been reported to be blurred in response to coloured stimuli ${ }^{30}$ with reduced colour fusion times. ${ }^{31}$ This response indicates loss of acuity of perception of monochromatic contours, but there is little evidence to date that colour vision is significantly affected in MSA. Consistent with this suggestion, abnormal visual evoked potentials (VEP) using coloured stimuli have not been reported. ${ }^{32}$ There have been few studies of visual field defects in patients with any parkinsonian syndrome; a consequence of the difficulties of testing patients with a movement disorder. A case of MSA has been reported, however, with enlarged blind spots but with no central scotoma. ${ }^{33}$ Some patients with neurodegenerative disease, in which impairment in VA have been demonstrated, also exhibit impairment of stereopsis as measured by random-dot stereograms ${ }^{34,35}$ but there is currently little information available for the parkinsonian syndromes.

\section{Eyelids and associated structures}

Given the autonomic dysfunction present in many MSA patients, problems with dry eye, blink reflex, and blepharospasm would be anticipated in MSA. Although not specifically observed in MSA, PD patients are prone to dry eye as a result of a combination of autonomic dysfunction and motor symptoms affecting blink rate. ${ }^{36}$ In addition, overall tear function abnormalities are significantly more common in PD including meibomian gland dysfunction. ${ }^{36}$

Eyelid reflexes are impaired in parkinsonian syndromes, especially PSP $^{2}$ including the 'acoustic blink reflex' and 'acoustic startle reflex', which are responses to a 
sudden and unexpected sound involving a brief closing of the eye. In a 65-year-old female patient with MSA, blink reflexes were poor with impaired R2 response and enhanced recovery cycle which could be attributable to increased excitability of brain stem interneurons. ${ }^{37}$

Blepharospasm is common in parkinsonian syndromes and more prevalent in 'atypical' parkinsonism such as PSP and MSA. ${ }^{38,39}$ In addition, early and prominent blepharospasm in untreated patients with parkinsonism should raise a suspicion of the presence of MSA, PSP, or CBD. ${ }^{40}$

\section{Eye movement}

Eye movement problems including those of the eyelids are an important feature of the parkinsonian syndromes in general. For example, spontaneous and voluntary eyelid mobility are often abnormal in PSP but are usually less affected in PD and MSA. ${ }^{41}$ Abnormal ocular fixation, however, may occur in a significant proportion of MSA patients. $^{42}$

In a patient with MSA, in which a combination of cerebellar and parkinsonian symptoms were present, the typical eye movement problems of PD were evident, viz., abnormal saccadic and smooth pursuit eye movements ${ }^{43}$, in combination with a vertical optokinetic nystagmus. ${ }^{44}$ By contrast, smooth pursuit and saccadic movements in the vertical direction were only slightly affected. Saccadic intrusions are continual movements that change position of the gaze during attempted fixation, and are often observed in MSA and other parkinsonian syndromes such as PSP. ${ }^{45,46}$ Hence, in a recent study of oculomotor function in thirty MSA patients ${ }^{46}$, excessive square-wave jerks were observed in 21/30 patients, a mild supranuclear gaze palsy in $8 / 30$ patients, a gaze-evoked nystagmus in 12/30 patients, a positioning down-beat nystagmus in 10/25 patients, mild-moderate saccadic hypometria in 22/30 patients, impaired smooth pursuit movements in 28/30 patients, and reduced vestibulo-ocular reflex (VOR) suppression in 16/24 patients. By contrast, whereas convergence problems are likely to be observed in PD and some DLB patients, there is little evidence that they occur in MSA. ${ }^{47}$ 
The VOR is a reflex eye movement that stabilizes images on the retina during movements of the head. This is achieved by the brain inducing an eye movement in the opposite direction to that of the head. It can be tested by the 'rapid head impulse test' in which the head is rapidly moved to the side. Normally the eyes will continue to fixate in the same direction but in MSA, no compensatory eye movement is apparent. The 'gain' of the VOR is the ratio of the change in eye angle to head angle during a head turn. If the gain is impaired (ratio not equal to unity), head movements result in image motion on the retina and blurred vision. The gain of the VOR in the dark may be cancelled by fixation. Both MSA and PSP patients show this cancellation compared with both normal subjects and patients with $\mathrm{PD}^{42}$, a response which could be related to cerebellar dysfunction. VOR can result in oscillopsia in some patients with MSA. ${ }^{29}$

\section{Pupil reactivity}

Examination of the pupil is an opportunity to detect disturbances in autonomic function in MSA. ${ }^{48}$ Infra-red pupil pupillography can be used to measure several aspects of pupillary function such as pupil diameters in the light and dark, the light reflex response, and pupil reactions associated with the administration of pharmacological agents. ${ }^{48}$ In one study of pupil reactivity in MSA, about a quarter of patients tested had abnormal pupils and in the majority, the defects were bilateral and symmetrical. Pupil defects were not usually apparent to the patients themselves or to their physicians. In addition, in normal subjects, pupil size often increases in response to stress but no such change is apparent in MSA. ${ }^{49}$ 'Horner's syndrome' (abnormally small pupil diameter) has rarely been observed, although such a change has been reported in one case of MSA. ${ }^{50}$

\section{Psychophysics}

Contrast sensitivity is affected in PD, at least in a proportion of patients ${ }^{51,52}$, but there is little evidence for a similar effect in MSA. For example, interocular differences in spatial contrast sensitivity have been observed in idiopathic PD but not in MSA. ${ }^{53}$ 
Visual spatial contrast threshold and suprathreshold contrast match to high-contrast sinewave gratings have been compared in PD and MSA patients and control subjects. ${ }^{54}$ The response of PD patients was affected compared with controls but this was not the case in MSA. Visuo-spatial function may therefore be largely spared in MSA. ${ }^{55}$

\section{Evoked potentials}

Evoked responses to motor stimuli have been reported to be normal in MSA. ${ }^{56}$ Nevertheless, although significant effects on the electroretinogram (ERG) and VEP have been found in PD and PSP, the results reported in MSA have been more controversial. For example, the pattern reversal ERG to a coloured stimulus was recorded in both PD and MSA. ${ }^{32}$ Although reduced amplitude and increased latency of evoked components were observed in PD, especially when a blue-yellow horizontal grating was used as a stimulus, no such effects were observed in MSA. ${ }^{32} \mathrm{~A}$ negative ERG, however, has been observed in some patients with MSA-C. ${ }^{40}$ Similarly, using a horizontal sinusoidal grating, there were no effects on the VEP in MSA although effects were demonstrated in PD. ${ }^{53}$ Significantly less impairment has also been observed in MSA to a full-field flash stimulus compared to PD and PSP. ${ }^{57}$ Nevertheless, in some studies, VEP abnormalities have been reported in MSA with no detectable differences between the MSA-C and MSA-P subtypes. ${ }^{56}$

\section{Event-related evoked potentials}

There have been a number of studies of event-related potentials in MSA, which elicit the 'P300' evoked response, believed to reflect orientation, attention, stimulus evaluation, and memory. These evoked responses may be associated with neural activity in the temporal lobe, prefrontal lobe, limbic system, and thalamus. ${ }^{58}$ In one of the first studies of event-related potentials in $\mathrm{MSA}^{59}$, it was observed that the P300, elicited using visual stimuli, could be recorded even when a severe motor disability was present. In addition, reaction times to rare target stimuli were significantly decreased in PD and MSA compared with PSP. ${ }^{58}$ Moreover, Kamitani et al. ${ }^{60}$ studied event related potentials in both MSA-C and MSA-P, no significant change being found in the latency of the N1 or N2 components in either disease subtype suggesting 
that the early stages of visual processing were preserved. Nevertheless, the P3a peak was less easy to distinguish and there were decreases in amplitude of the response in both MSA subtypes. By contrast, the P3b component was only affected in the MSA-C subtype and exhibited both increased latency and reduced amplitude. In a further study $^{61}$, the N2 component was studied during selective attention to colour and was significantly delayed although the N1 component was clearly evident. The data suggest that the colour discrimination process may be impaired during selective attention in MSA. The reduced size of the pons, claustrum, perisylvian area, and the deep cerebral gray matter may be implicated in these responses.

\section{Complex visual functions}

Speech abnormalities are common in all parkinsonian syndromes including MSA, in which there is often a mixed dysarthria (problems in articulating speech) with ataxic and spastic elements. ${ }^{62}$ Maximum phonation time and reading speed are also affected but to a lesser extent than in PSP. ${ }^{62}$ The 'room-tilt illusion', a transient visuo-spatial perception problem, has been observed in $\mathrm{MSA}^{63}$, and may be caused by an erroneous cortical mismatch of visual and vestibular information.

Sleep disorders and excessive daytime sleeping are common in MSA $^{64}$ and may be caused by pathology affecting the regulation of the sleep/awake transition and the respiratory function. Sleep behavioural disorder episodes decrease in frequency but sleep becomes increasingly abnormal as the disease develops. ${ }^{65}$ There is also 'idiopathic rapid eye movement' (iREM) disorder in MSA, characterised by abnormal behaviour during sleep accompanied with rapid eye movements, and which probably result from neuronal losses in the locus caeruleus and substantia nigra. ${ }^{66}$ There is also loss of neurons in the pedunculopontine and laterodorsal tegmental nuclei in MSA and $\mathrm{DLB}^{67}$ but these changes are unlikely to be the cause of the REM sleep disorder. So common is iREM in the parkinsonian syndromes generally, that it is often regarded as a strong indicator of the presence of a parkinsonian-type disorder. Visual hallucinations, unrelated to medication, are rare in MSA compared with the other parkinsonian syndromes and especially $\mathrm{PD}^{68}$ and may occur in only about 5-9\% of patients. $^{11}$ 


\section{Differential diagnosis}

The parkinsonian syndromes exhibit overlapping signs and symptoms and therefore, can be difficult to distinguish with confidence. There are a number of general clinical features, however, that can help to distinguish MSA from PD, PSP, DLB, and CBD. First, patients with MSA often exhibit a combination of extrapyramidal, cerebellar, pyramidal, and autonomic dysfunction signs, many of which can be seen in other parkinsonian disorders. Second, MSA patients often show early autonomic problems, including those associated with pupil reactivity and urological failure, signs which are less likely to be observed in the other disorders. ${ }^{8}$ Third, imaging studies may also help to distinguish these disorders. Hence, PSP may be separated from MSA because it often exhibits atrophy of the midbrain/tegmentum on magnetic resonance imaging (MRI) ${ }^{69}$ Fourth, regional cerebral blood flow (rCBF) in the left frontal eye field is lower in PD compared with other parkinsonian syndromes such as MSA. Fifth, growth hormone responses may also help to distinguish MSA-P from PD, responses being generally greater in MSA than in the other syndromes. Specifically, the response to clonidine is greater than that to arginine, but combined response to both agents may have even greater sensitivity and specificity. ${ }^{70}$

Where visual signs and symptoms are present, they can provide additional information for differential diagnosis. There is little current information relating each visual symptom to stage of the disease. However, pupil reactivity due to autonomic dysfunction, is likely to be an early feature of MSA in a proportion of cases ${ }^{40}$ while most other features are more likely to develop later in the disease. Hence, the presence of visual hallucinations, unrelated to medication, is rare in MSA compared with PD and DLB and their presence can often exclude MSA as a possible diagnosis. ${ }^{71}$ In addition, there is greater retinal pathology in $\mathrm{PD}$, which results in significant defects in contrast sensitivity and in the early components of the VEP, which are not likely to be present in MSA. Moreover, colour VEPs are affected in PD but not in MSA. ${ }^{32}$ Nevertheless, where defects in the VEP are observed in MSA, they are more likely to involve event-related evoked potential and components such as the P300. ${ }^{58,59}$ 
Eye movement problems, especially those related to oculomotor dysfunction, are an important feature of MSA. Nevertheless, many of the visual signs and symptoms associated with eye movement in MSA are controversial and may not occur in all patients. Some authors believe that oculomotor anomalies are not present in MSA consistently enough to be useful in differential diagnosis. ${ }^{72}$ Additional features which may be useful include fixation, which is abnormal in a significant proportion of patients with PSP and MSA but less so in $\mathrm{PD}^{42}$, eyelid mobility which is abnormal in PSP but more likely to be normal in PD and MSA ${ }^{41}$, and the slowing of saccadic movements, especially in the vertical direction, which occurs most significantly in PSP. $^{73}$ In addition, patients with PSP exhibit large saccadic intrusions which are visible in the absence of a fixation target while in MSA there are increasing saccadic intrusions with or without a visible target. ${ }^{74}$ Eye movements recorded during sinusoidal tracking by video-oculography ${ }^{75}$ show that in MSA, saccades correct for position error ('catch-up saccades') while in PD, saccades are directed towards future target positions ('anticipatory saccades'). Fixation instability is a significant feature of PSP and MSA and is less often seen in PD and DLB.

Anderson et al. $^{46}$ have published a list of 'red flag' criteria which are strongly suggestive of a diagnosis of MSA and which include excessive square-wave jerks, mild to moderate hypometria of saccades, impaired VOR, and nystagmus. By contrast, if clinically slow saccades are present together with a moderate to severe vertical gaze palsy, the diagnosis is more likely to be one of the other parkinsonian syndromes, most probably PSP. However, caution in the use of many of these criteria has been advised in the diagnosis of MSA. ${ }^{76}$ An additional feature that may be useful in diagnosis is that in $\mathrm{PD}$, there is often lateral jaw movement ipsilateral to the direction of horizontal gaze (ocular jaw synkinesia) ${ }^{77}$ while its absence would suggest MSA.

\section{MSA and the optometrist}

Middle-aged to elderly patients, who have not been diagnosed with MSA, may exhibit visual signs and symptoms suggestive of one of the parkinsonian-type disorders. The most important visual signs of MSA include oculomotor dysfunction and pupil reactivity, but with relatively preserved primary vision. Hence, a patient 
with a combination of these features, together with autonomic or cerebellar dysfunction, should be referred for neurological examination.

The following tests and procedures may be useful in identifying the visual problems of a patient with MSA. It is particularly important to carry out the full examination including ocular health assessment. It should be noted, however, that such patients may have problems in describing their symptoms and are often 'tolerant' of their visual problems. ${ }^{78}$ Hence, clinical examination requires sensitivity to the physical and mental state of the patient and the problems involved in the parkinsonian syndromes in general have been described in detail by Naylor. ${ }^{79}$ Subsequently, several additional tests may be helpful such as regular assessment of the general health of the exterior eye as dry eye, impaired blink reflex, and blepharospasm may be present. In addition, various aspects of oculomotor function should be assessed where possible as squarewave jerks, supranuclear gaze palsy, a gaze-evoked nystagmus, a positioning downbeat nystagmus, saccadic hypometria, impaired smooth pursuit movements, and reduced vestibulo-ocular reflex (VOR) suppression may all be present.

Management of the disease to deal with problems of walking, movement, daily tasks, and speech problems is important in MSA. Optometrists can work in collaboration with the patient and health care providers to identify and manage the specific visual deficits of the patient. Management may involve physiotherapy to maintain mobility and prevent contractures, gait-training to improve mobility and decrease the risk of falls, and the use of social workers and occupational therapists to oversee the provision of equipment and home adaptation. Poor vision assumes a greater importance as mobility decreases with age. Hence, nuclear cataract can increase the risk of falling independent of age ${ }^{80}$, poor binocular vision is often associated with social isolation ${ }^{81}$, and unilateral visual loss with reductions in quality of life. ${ }^{82}$ These problems are likely to be even greater in patients with MSA who can potentially develop a range of visual problems during the course of the disease, many of which are common to other types of parkinsonian syndromes. ${ }^{1-3}$ Visual deficits in parkinsonian-type syndromes are often important in influencing overall motor function. Hence, identifying and correcting the visual problems as far as possible can significantly reduce the chances that MSA patients will suffer serious falls. In addition, the external eye should be monitored as blepharospasm and possibly dry eye 
may be present. Hence, in a 65-year-old female with MSA, the patient was found to be functionally blind due to blepharospasm. ${ }^{37}$ These symptoms should be investigated as blepharospasm is an essentially treatable visual feature of parkinsonian syndromes such as MSA.

Some of the visual problems observed in these patients may be adverse reactions to treatment. Side effects may occur relatively rapidly at the beginning of a treatment, or if medication has changed, but can also occur after a long latent period. It is important that those symptoms due to adverse reactions are separated from those due to the disease process itself. If ocular side effects are identified in a patient with MSA and become severe, then it is essential that these are monitored and the patient referred back to their physician for further clinical assessment.

\section{Conclusions}

Multiple system atrophy (MSA) is a complex syndrome in which patients exhibit a variety of signs and symptoms including those of parkinsonism, ataxia, and autonomic dysfunction. The most important visual signs of MSA include oculomotor and pupil reactivity dysfunction, but with relatively preserved primary vision. Nevertheless, the exact presentation of MSA is highly variable and patients can be visually asymptomatic. It is difficult to separate MSA from other disorders with parkinsonism such as PD and PSP. If ocular signs and symptoms are present, then they may aid differential diagnosis. Typical ocular features of MSA include excessive square-wave jerks, mild to moderate hypometria of saccades, impaired vestibularocular reflex (VOR), and nystagmus. Less typical of MSA are slowing of saccades and the presence of vertical gaze palsy. Management of the disease to deal with problems of walking, movement, daily tasks, and speech problems is important in MSA. The optometrist can play an important role in this management both in ensuring that visual problems are corrected as far as possible and in referring patients to relevant health professionals. There is a more specific role for the optometrist in correcting vision to prevent falls and to monitor the anterior eye to prevent dry eye and control blepharospasm.

\section{References}


1. Armstrong RA. Visual signs and symptoms of Parkinson's disease. Clin Exp Optom 2008; 91: 129-138.

2. Armstrong RA. Visual signs and symptoms of progressive supranuclear palsy. Clin Exp Optom 2011; 95: 150-160.

3. Armstrong RA. Visual signs and symptoms of dementia with Lewy bodies: Clin Exp Optom 2012; 94: 621-630.

4. Armstrong RA, Cairns NJ, Lantos PL. A quantitative study of the pathological lesions in neocortex and hippocampus of 12 patients with corticobasal degeneration. Exp Neurol 2000; 163: 348-356.

5. Probst-Cousin S, Kayser C, Heuss D, Neundorfer B. Thirty years of multiple system atrophy concept: Review and survey of multiple system atrophy. Fortschritte Neurol Psych 2000; 68: 25-36.

6. Lantos PL. Cellular pathology of multiple system atrophy: a review. J Neurol Neurosurg Psychiatr 1994; 57: 129-13.

7. Bower JH, Maraganore DM, McDonnell K, Rocca WA. Incidence of progressive supranuclear palsy and multiple system atrophy in Olmstead County, Minnesota, 1976-1990. Neurology 1997; 49: 1284-1288.

8. Wenning GK, Tison F, Ben-Shlomo Y, Daniel SE, Quinn NP. Multiple system atrophy: a review of 203 pathologically proven cases. Move Disord 1997; 12: 133147.

9. Ben-Shlomo Y, Wenning GK, Tison F, Quinn NP. Survival of patients with pathologically proven multiple system atrophy: A meta analysis. Neurology 1997; 48: 384-393. 
10. Sakakibara R, Hattori T, Uchiyama T, Kita K, Asahina M, Suzuki A, Yamamshi T. Urinary dysfunction and orthostatic hypotension in multiple system atrophy: which is the more common and earlier manifestation? J Neurol Neurosurg Psych 2000; 68: 65-69.

11. Stefanova N, Bucke P, Duerr S. Wenning GK. Multiple system atrophy: an update. Lancet Neurology 2009; 8: 1172-1178.

12. Ehrt V, Brieger P, Broich K, Marneros A. (1999) Psychotic symptoms as initial manifestation of a multiple system atrophy. Fortsch der Neurol Psych 1999; 67: 104107.

13. Gilman S, Low PA, Quinn N, Albanese A, Ben-Schlomo Y, Fowler CJ, Kaufmann H, Klockgether T, Lang AE, Lantos PL, Livan I, Mathias CJ, Oliver E, Roberstson D, Schatz I, Wenning GK. Consensus statement on the diagnosis of multiple system atrophy. J Auto Nerv Syst 1998; 74: 189-192.

14. Gilman S, Wenning GJK, Low PA, Brooks DJ, Mattias CJ, Trojanowski JQ, Wood NW, Colosima C, Durr A, Fowler CJ, Kaufmann H, Klockgether T, Lees A, Poese W, Quinn N, Revesz T, Roberstson D, Sandroni T, Seppi K, Vidailhet M. Second consensus statement on the diagnosis of multiple system atrophy. Neurology 2008; 71: 670-676.

15. Horimoto Y, Aiba I, Yasuda T, Ohkawa Y, Katayama T, Yokokawa Y, Goto A, Ito Y. Cerebral atrophy in multiple system atrophy by MRI. J Neurol Sci 2000; 173: 109-112.

16. Konogaya M, Sakai M, Matsuoka Y. Konogaya Y, Hashzume Y. Multiple system atrophy with remarkable frontal lobe atrophy. Acta Neuropathol 1999; 97: 423-428.

17. Wakabayashi K, Ikeuchi T, Ishikawa A, Takahashi H. Multiple system atrophy with severe involvement of the motor cortical areas and cerebral white matter. $J$ Neurol Sci 1998; 156: 114-117. 
18. Tsuchiya K, Watabiki S, Sano M, Iobe H, Shiotsu H, Taki K, Hashimoto K. Distribution of cerebellar cortical lesions in multiple system atrophy: a topographic neuropathological study of three autopsy cases in Japan. J Neurol Sci 1998; 155: 8085.

19. Papp MI, Kahn JE, Lantos PL. Glial cytoplasmic inclusions in the CNS of patients with multiple system atrophy (striatonigral degeneration, olivopontocerebellar atrophy, and Shy-Drager syndrome). J Neurol Sci 1989; 94: 79100.

20. Dickson DW, Liu WL, Liu WK, Yen SH. Multiple system atrophy: a sporadic synucleinopathy. Brain Pathol 1999; 9: 721-732.

21. Spillantini MG, Crowther RA, Jakes R, Cairns NJ, Lantos PL, Goedert M. Filamentous alpha-synuclein inclusions link multiple system atrophy with Parkinson's disease and dementia with Lewy bodies. Neurosci Lett 1998; 251: 205208.

22. Wenning GK, Quinn NP. Multiple system atrophy. Baillieres Clin Neurol 1997; 6: $187-204$.

23. Ozawa T, Takano H, Onodero O, Kobayashi H, Ikeuchi T, Koide R, Okizumi K, Shimohata T, Wakabayashi K, Takahashi H, Tsuji S. No mutation in the entire coding region of the alpha-synuclein gene in pathologically confirmed cases of multiple system atrophy. Neurosci Lett 1999; 270: 110-112.

24. Hashida H, Goto J, Zhao ND, Takahashi N, Hirai M, Kanazawa I, Sakaki Y. Cloning and mapping of ZNF231, a novel brain-specific gene encoding neuronal double zinc finger protein whose expression is enhanced in a neurodegenerative disease multiple system atrophy. Genomics 1998; 54: 50-58.

25. Sasaki H, Emi M, Iijima H, Ito N, Sato H, Yabe I, Kato T, Utsumi J, Matsubara K. Copy number loss of (Src homology 2 domain containing) transforming protein 2 (SHC2) gene: discordant loss in monozygotic twins and frequent loss in patients with 
multiple system atrophy. Mole Brain 2011; 4: Article 24. DOI:10:1186/1756-6606-424.

26. Cairns NJ, Atkinson PF, Kovacs T, Lees AJ, Daniel SE, Lantos PL. Apolipoprotein E e4 allele frequency in patients with multiple system atrophy. Neurosci Lett 1997; 221: 161-164.

27. Ozawa T, Revesz T, Paviour D, Lees AJ, Quinn N, Tada M, Kakita A, Onodera O, Wakabayashi K, Takajashi H, Nishizawa M, Holton, JL. Difference in MSA phenotype distribution between populations: Genetics or Environment. J Park Dis 2012; 2: 7-18.

28. Mathias CJ, Senard JM, Braune S, Watson L, Aragashi A, Keeling JEA, taylor MD. L-threo-dihydroxyphenylserine (L-threo-DOPS; droxidopa) in the management of neurogenic orthostatic hypotension: a multi-national, multi-center, dose-ranging study in multiple system atrophy and pure autonomic failure. Clin Auton Res 2001; 11: $235-242$.

29. Guzulla J, Benavente MI. Improvements in the symptoms of olivopontocerebellar atrophy with gabapentin. Rev de Neurol 2005; 40: 285-288.

30. Price MJ, Feldman RG, Adelberg D, Kayne H. Abnormalities in colour vision and contrast sensitivity in Parkinson's disease. Neurology 1992; 42: 887-890.

31. Buttner T, Kuhn W, Klotz P, Steinberg R, Voss L, Bulgaru D, Przuntek H. Disturbance of colour perception in Parkinson's disease. J Neural Transm 1993; 6: 11-15.

32. Sartucci F, Orlandi G, Bonuccelli U, Borghetti D, Murri L, Orsini C, Domenica L, Porciatti V. Chromatic pattern-reversal electroretinogram (ChPERG) are spared in multiple system atrophy compared with Parkinson’s disease. Neurol Sci 2006; 26 : 395-401. 
33. Barnes CS, Yan J, Wilmot GR. A negative electroretinogram (ERG) in a case of probable multiple system atrophy (MSA). Doc Ophthalmol 2009; 118: 247-256.

34. Cronin-Golomb A, Corkin S, Rizzo JF, Cohen J, Growden JH Banks KS. Visual dysfunction in Alzheimer's disease: relation to normal ageing. Ann Neurol 1991; 29: 41-52.

35. Kiyosawa M, Bosley TM, Chawluk J, Jamieson D, Schatz NJ, Savino PJ, Sergott RC, Reivich M, Alavi A. Alzheimer's disease with prominent visual symptoms; clinical and metabolic evaluation. Ophthalmology 1989; 96: 1077-1085.

36. Tamer C, Melek IM, Dumen T, Oksuz H. Tear film tests in Parkinson's disease patients. Ophthalmology 2005; 112: 1795-1800.

37. Kagohashi M, Okuma Y, Fujishima K, Kitada Y, Mizuno Y. Blepharospasm associated with multiple system atrophy: a case report and review of the literature. Parkin Rel Disord 2004; 10: 169-171.

38. Leon-Sarmiento FE, Bayona-Prieto J, Gomez J. Neurophysiology of blepharospasm and multiple system atrophy: clues to pathophysiology. Parkin Rel Disord 2005; 11: 199-201.

39. Rana AO, Kabir A, Dogu O, Patel A, Khondker S. Prevalence of blepharospasm and apraxia of eye opening in patients with parkinsonism, cervical dystonia, and essential tremor. Eur Neurol 2012; 68: 318-321.

40. Tolosa E, Compta Y. Early and prominent blepharospasm in untreated patients with parkinsonism should raise suspicion of progressive supranuclear palsy, multiple system atrophy, or corticobasal degeneration. J Neurol 2005; 253: 7-13.

41. Valls-Sole J, Valldeoriola F, Tolosa E, Marti MJ. Distinctive abnormalities of facial reflexes in patients with progressive supranuclear palsy. Brain 1997; 120: 1877-1883. 
42. Rascol O, Sabatini U, Fabre N, Senard JM Simonett-Amoreau M, Montastruc JL, Clanet M, Rascol A. Abnormal vestibulo-ocular cancellation in multiple system atrophy and progressive supranuclear palsy but not in Parkinson's disease. Move Disord 1995; 10: 163-170.

43. Shibasaki H, Tsuji S, Kuroiwa Y. Oculomotor abnormalities in Parkinson's disease. Arch Neurol 1979; 36: 360-364.

44. Neetens A, Vanderende P, Smet H, Martin JJ. Disturbed vertical optokinetic nystagmus: possible sign of striatonigral pathway involvement. NeuroOphthalmology 1991; 11: 141-149.

45. Altiparmak UE, Eggenberger E, Coleman A, Condon K. The ratio of square wave jerk rates to blink rate distinguishes progressive supranuclear palsy from Parkinson's disease. J Neuro-ophthalmol 2006; 26: 257-259.

46. Anderson T, Luxon L, Quinn N, Daniel S, Marsden CD, Bronstein A. Oculomotor function in multiple system atrophy: clinical and laboratory features in 30 patients. Move Disord 2008; 23: 977-984.

47 Corin MS, Elizan TS, Bender MB. Oculomotor function in patients with Parkinson's disease. J Neurol Sci 1971; 15: 251-265.

48. Bremner F, Smith S. Pupil findings in a consecutive series of 150 patients with generalised autonomic neuropathy. J Neurol Neurosurg Psych 2006; 77: 1163-1168.

49. Kuroda M, Fukura H, Saruki N, Yoshikawa D, Morita T, Goto F. Vecuronium dose requirement and pupillary response in a patient with olivopontocerebellar atrophy (OPCA). Can J of Anaesthesia 1998; 45: 979-984.

50. Smith SA, Smith SE. Bilateral Horner's syndrome: detection and occurrence. $J$ Neurol Neurosurg Psychiatr 1999; 66: 48-51. 
51. Bulens C, Meerwaldt JD, Van der Wildt GJ, Van Deursen JBP. Effect of levodopa treatment on contrast sensitivity in Parkinson's disease. Ann Neurol 1987; 22: 365-369.

52. Hutton JT, Morris JL, Elias JW. Levodopa improves spatial contrast sensitivity in Parkinson's disease. Arch Neurol 1993; 50, 721-724.

53. Delalande I, Hache JC, Forzy G, Bughin M, Benhadjali J, Destee A. Do VEP and spatialtemporal contrast sensitivity help to distinguish idiopathic Parkinson's disease and multiple system atrophy. Move Disord 1998; 13: 446-452.

54. Van Elst LT, Greenlee MW, Foley JM, Lucking CH. Contrast detection discrimination and adaptation in patients with Parkinson's disease and multiple system atrophy. Brain 1997; 120: 2219-2228.

55. Bak TH, Caine D, Hearn VC, Hodges JR. Visuospatial functions in atypical parkinsonian syndromes. J Neurol Neurosurg Psychiatr 2006; 77: 454-456.

56. Abele M, Schulz JB, Burk K, Topka H, Dichgans J, Klockgetter T. Evoked potentials in multiple system atrophy (MSA). Acta Neurol Scand 2000; 101: 111-115.

57. Langheinrich T, van Elst LT, Lagreze WA, Bach M Lucking CH, Greenlee MW. Visual contrast response functions in Parkinson's disease: evidence from electroretinogram, visually evoked potentials and psychophysics. Clin Neurophysiol 2000; 111: 66-74.

58. Wang LH Kuroiwa Y, Kamitani T, Li M, Takahashi T, Suzuki Y, Shimamura M, Hasegawa O. Visual event-related potentials in progressive supranuclear palsy, corticobasal degeneration, striatonigral degeneration and Parkinsons's disease. $J$ Neurol 2000; 247: 356-363.

59. Koga Y, Nagata K, Hirata K. Hypothesis on the P300 generators based on visual P300 results in neurological disorders. Brain Topog Today Int Cong series 1997; 1147: 354-357. 
60. Kamitani T, Kuroiwa T, Wang LH, Li M, Suzuki Y, Takahashi T, Ikegami T, Matsubara S. Visual event-related potential changes in two subtypes of multiple system atrophy, MSA-C and MSA-P. J Neurol 2002; 249: 975-982.

61. Kamitani T, Kuroiwa Y. Visual event-related potential changes in multiple system atrophy: delayed N2 latency in selective attention to a color task. Park Dis Rel Disord 2009; 15: 36-40.

62. Sachin S, Shukla G, Goyal V, Singh S, Aggrawal V, Guresh-Kumar, Behari M. Clinical speech impairment in Parkinson's disease, progressive supranuclear palsy, and multiple system atrophy. Neurology India 2008; 56: 122-126.

63. Sierra-Hidalgo F, de Pablo-Fernandez E, Martin AHS, Corresa-Callero E, Herreros-Rodriguez J, Romero-Munoz JP, Martin-Gil L. Clinical and imaging features of the room tilt illusion. J Neurol 2012; 259: 2555-2564.

64. Ghorayeb I, Biolac B, Tison F. Sleep disorders in multiple system atrophy. $J$ Neural Transm 2005; 112: 1669-1675.

65. Vetrugno R, Alessandria M, D’Angelo R, Plazzi G, Provini F, Cortelli P, Montagno P. Status dissociatus evolving from REM sleep behavioural disorder in multiple system atrohy. Sleep Med 2009; 10: 247-252.

66. Turner RS. Idiopathic rapid eye movement sleep behaviour disorder is a harbinger of dementia with Lewy bodies. J Ger Psych Neurol 2002; 15: 195-199.

67. Schmeichel AM, Buchhalter LC, Low PA, Parisi JE, Boeve BW, Sandroni P, Benarroch EE. Mesopontine cholinergic neuron involvement in Lewy body dementia and multiple system atrophy. Neurology 2008; 70: 368-373.

68. Bertram K, Williams DR. Visual hallucinations in the differential diagnosis of parkinsonism. J Neurol, Neurosurg and Psych 2012, 83, 448-452. 
69. Kurata T, Kametaka S, Ohta Y, Morimoto N, Deguci S, Deguchi K, Ikeda Y, Takao Y, Ohta T, Manabe Y, Sato SH, Abe K. PSP as distinguished from CBD, MSA-P and PD by clinical and imaging differences at early stage. Int Med 2011; 50: 2775-2781.

70. Zhang KZ, Zeng YY, Song CJ, Fu Y, Wan Q. The comparison of clonidine, arginine and both combined: a growth hormone stimulation test to differentiate multiple system atrophy from idiopathioc Parkinson's disease. J Neurol 2010; 257: 1486-1491.

71. Kumbler E, Kornhuber M. Delusional parasitosis in multiple system atrophy. Nervenarzt 2002; 73: 380-383.

72. Pierrot-Deseilligny C, Pivauld-Pechoux S. Contribution of oculomotor explotation for the etiological diagnosis of parkinsonian syndromes. Rev Neurol 2003; 159: S75-S81.

73. Rottack KG, Riley DE, DiScenna AO, Zivotofsky AZ, Leigh RJ. Dynamic properties of horizontal and vertical eye movements in parkinsonian syndromes. Ann Neurol 1996; 39: 368-377.

74. Pinnock RA, McGivern RC, Forbes R, Gibson JM. An exploration of ocular fixation in Parkinson's disease, multiple system atrophy and progressive supranuclear palsy. J Neurol 2010; 257: 533-539.

75. Pinkhardt EH, Kassubek J, Sussmuth S, Ludolph AC, Becker W, Jurgens R. Comparison of smooth pursuit eye movement deficits in multiple system atrophy and Parkinson’s disease. J Neurol 2009; 256: 1438-1446.

76. Fletcher WA. Eye signs in patients with parkinsonism: do they predict the development of multiple system atrophy. Nature Clin Prac 2008; 4: 540-541. 
77. Salazar G, Casas E, Oliveras D, Rando A, Sergio P. Ocular-jaw synkinesia in normal, Parkinson's disease, and multiple system atrophy subjects: Clinical and electrophysiological findings. Clin Neurophysiol 2010; 121: 94-97.

78. Koch JM, Datta G, Makdoom S, Grossberg, GT. Unmet visual needs of Alzheimer's disease patients in long-term care facilities. J Am Med Dir Assoc 2005; 6: 233-237.

79. Naylor RJ. The ocular effects of parkinsonism and its treatment. Optometry in Practice 2005; 6: 19-31.

80. McCarty CA, Fu CL, Taylor HR. Predictors of falls in the Melbourne visual impairment project. Aust NJ J Pub Health 2002; 26: 116-119.

81. West SK, Munoz B, Rubin GS, Schein OD, Bandeen-Roche K, Zeger S, German PS, Fried LP. Function and visual impairment in a population-based study of older adults. The SEE project. Salisbury Eye Evaluation. Invest Ophthalmol Vis Sci 1997; 38: 72-82.

82. Vu HT, Keeffe JE, McCarty CA, Taylor HR. Impact of unilateral and bilateral vision loss on quality of life. Br J Ophthalmol 2005; 89: 360-363. 
Table 1. Common signs and symptoms of multiple system atrophy (MSA)

Low blood pressure when standing up

High blood pressure when lying down

Impotence and loss of libido

Loss of bladder or bowel control

Rigidity of muscles

Unsteady gait and loss of balance

Reduction in perspiration, tears and saliva

Impaired control of body temperature

Constipation

Slurred speech

Visual disturbances

Irregular heartbeat

Impaired ability to swallow

Difficulty in controlling emotions

Agitated sleep

Impaired breathing at night 
Table 2. Summary of visual signs and symptoms in Multiple system atrophy (MSA)

\begin{tabular}{|c|c|c|}
\hline$\underline{\text { Ocular aspect }}$ & Change in MSA & $\underline{\text { Referen }}$ \\
\hline Primary vision & $\begin{array}{l}\text { Affected in other forms of parkinsonism } \\
\text { but little evidence in MSA }\end{array}$ & $1-3$ \\
\hline Colour vision & Unlikely to be affected & $30-32$ \\
\hline $\begin{array}{l}\text { Eyelids and } \\
\text { related structures }\end{array}$ & $\begin{array}{l}\text { May be dry eyes, impaired blink reflex, } \\
\text { and blepharospasm }\end{array}$ & $36-40$ \\
\hline Eyelid mobility & $\begin{array}{l}\text { Spontaneous and voluntary eyelid mobility } \\
\text { normal }\end{array}$ & 41 \\
\hline
\end{tabular}

Fixation

Abnormal in many patients

42

Convergence

No problems reported

47

Square-wave jerks

Present in a significant proportion of

patients

Supranuclear gaze Mild defect in some patients

46

palsy

Nystagmus

Gaze-evoked nystagmus in a proportion

46

of patients

Saccadic hypometria Mild to moderate

Smooth pursuit

Impaired in a significant number of

movement

patients

VOR

Reduced suppression in a proportion of 
Patients. May lead to oscillopsia

Pupil reactivity Abnormal pupils in many patients,

$48-50$

No response due to stress

Contrast sensitivity No evidence for impairment

Visuo-spatial

Largely unaffected

55

function

ERG

Normal colour pattern ERG

56

Negative ERG in some patients

40

VEP

Defects controversial

53

Normal

Event-related

Abnormal P300 in some patients

58-61

Sleep disorder

iREM in many patients

64-66

Reading speed Mildly affected

62

Visual hallucinations Rare, unrelated to medication

13,68

Abbreviations: ERG $=$ Electroretinogram, $\mathrm{iREM}=$ Idiopathic rapid eye movement sleep disorder, VEP = Visual evoked responses, VOR = Vestibulo-ocular reflex 


\section{Legends to figures}

Fig 1. Coronal section of the brain of a patient with multiple system atrophy (MSA) showing the small and discoloured putamen (Put). Other abbreviations: $\mathrm{CN}=$ Caudate nucleus, GP = Globus pallidus, $\mathrm{IC}=$ Internal capsule, $\mathrm{Th}=$ Thalamus

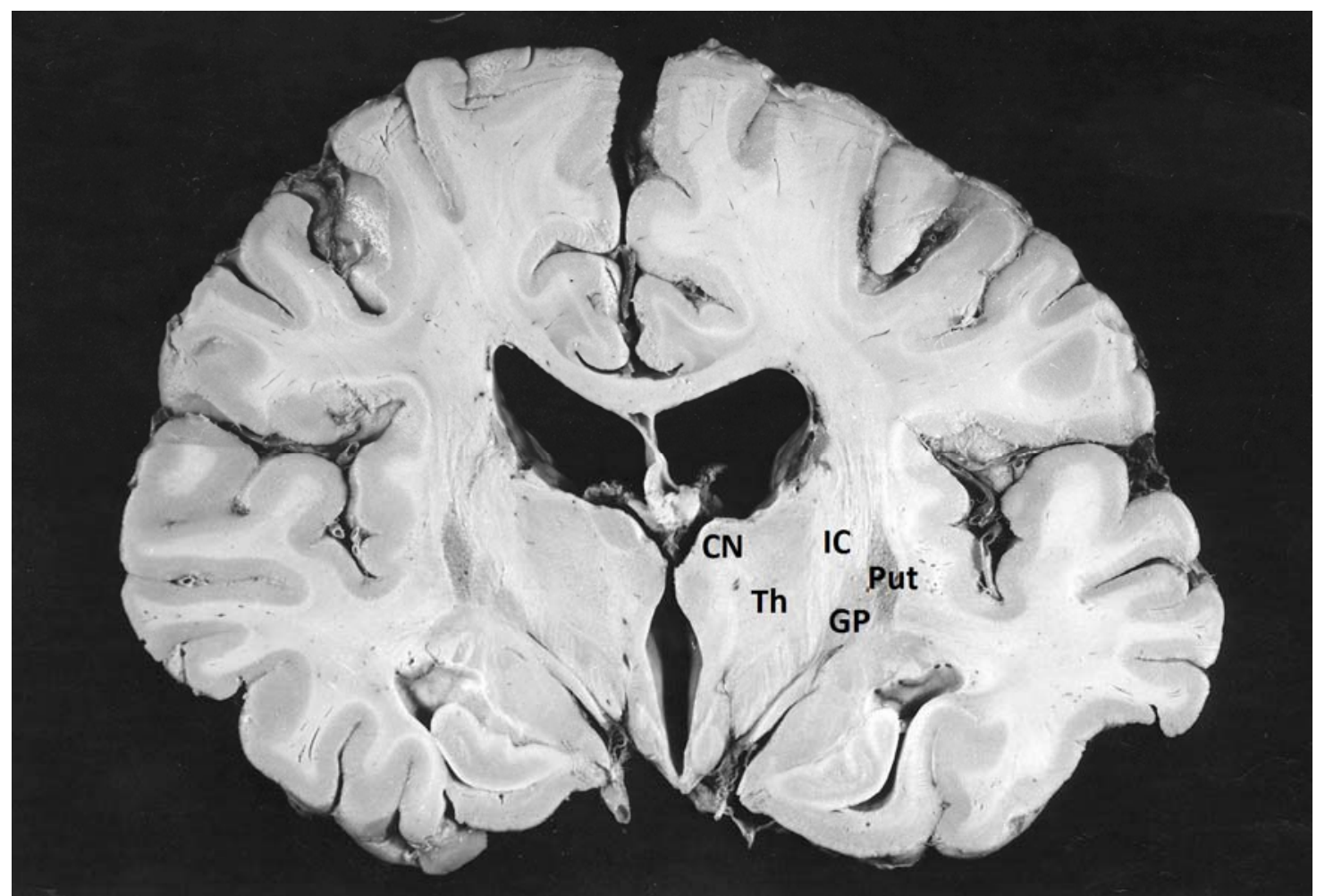


Fig 2. The areas of the brain affected by the pathology of multiple system atrophy (MSA) (shown in bold in large font) (SFC = Superior frontal cortex, OC = Occipital cortex, STG = Superior temporal gyrus, $\mathrm{PHG}=$ Parahippocampal gyrus, $\mathrm{HC}=$ Hippocampus, $\mathrm{CG}=$ Cingulate gyrus, $\mathrm{I} / \mathrm{C}=$ Insula/claustrum, $\mathrm{A}=$ Amygdala, $\mathrm{nB}=$ Nucleus basalis of Meynert, Put = Putamen, $\mathrm{CN}=$ caudate nucleus, $\mathrm{P}=$ Pulvinar of thalamus, $\mathrm{SN}=$ Substantia nigra, $\mathrm{R}=$ Raphe nuclei, $\mathrm{LC}=$ Locus careuleus, Other areas identified (in normal text and small font) (OG = orbital gyrus, GR = Gyrus rectus, $\mathrm{PC}=$ Parietal cortex, $\mathrm{DG}=$ Dentate gyrus, $\mathrm{Th}=$ Thalamus, $\mathrm{LGN}=$ Lateral geniculate nucleus, $\mathrm{Hy}=$ Hypothalamus, $\mathrm{VT}=$ Ventral tegmentum, Ce $=$ Cerebellum, MB = Mamillary bodies, GP = Globus pallidus) superimposed on a two-dimensional model of the brain based on that of WJH Nauta and M Feirtag (1986) Fundamental Neuroanatomy. WH Freeman \& Co. Hence the cerebral cortex is represented at the right of the diagram with the striatum (ST) and thalamus (Th) below. Midbrain and brain stem nuclei are at the left of the diagram

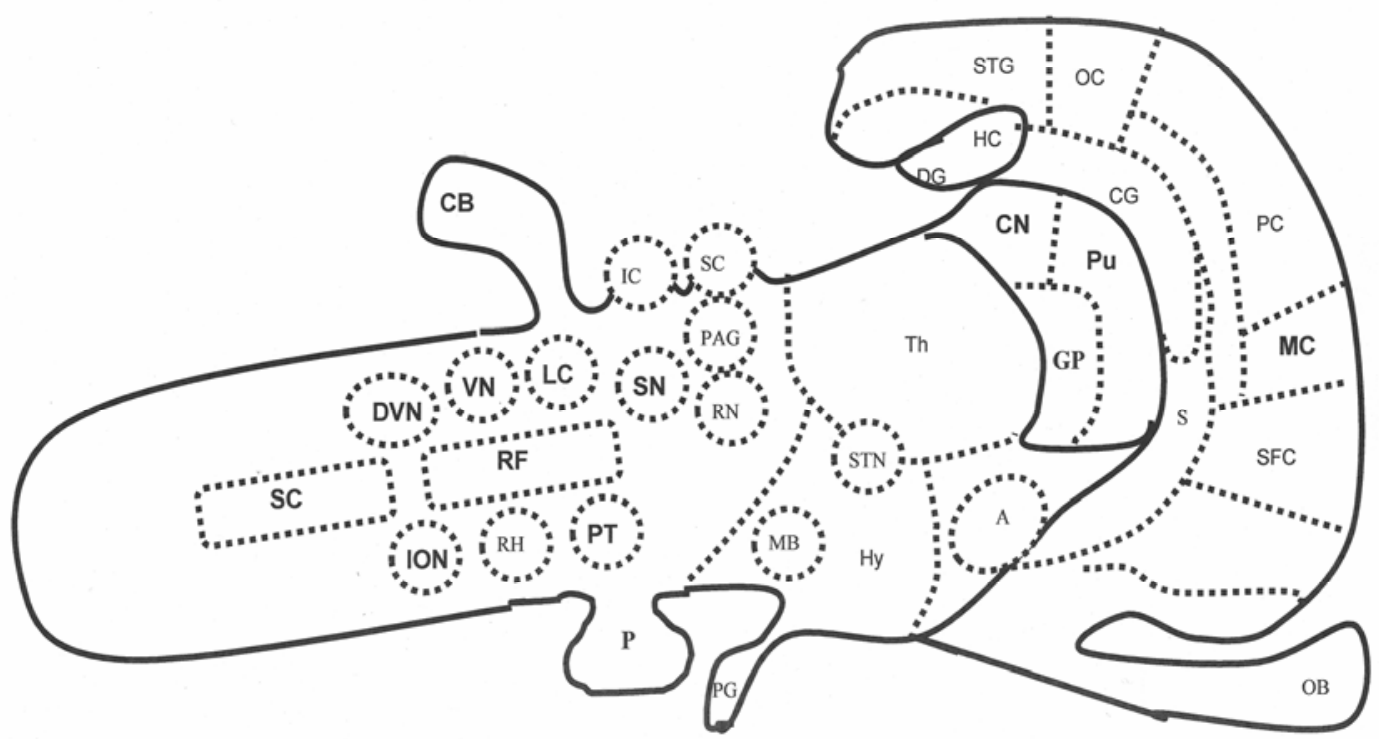


Fig 3. Mean density of glial cytoplasmic inclusions (GCI) in various brain regions in 10 cases of multiple system atrophy (MSA). The greatest densities of GCI are seen in basal ganglia including the putamen (PT), globus pallidus (GP), and substantia nigra (SN). Significant numbers of GCI are also observed in the pons (PN), inferior olivary nucleus (ION), and cerebellum hemisphere (CB). Relatively low densities of GCI are observed in the superior frontal gyrus (SFG), superior temporal gyrus (STG), inferior temporal gyrus (ITG), parahippocampal gyrus (PHG), and hippocampus (HC). Data from Armstrong et al. (2004). Error bars indicate SE of mean

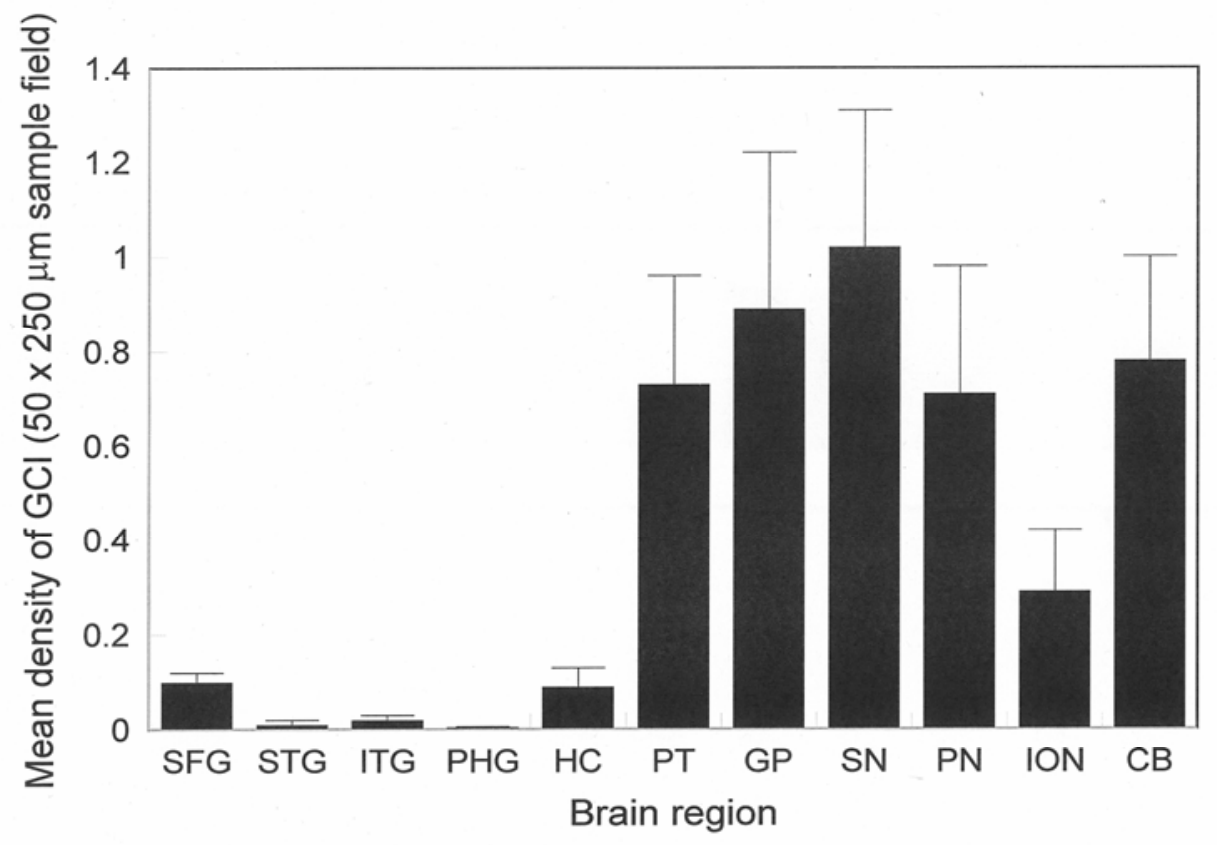


Fig 4. The major brain areas affected in multiple system atrophy (MSA) involved in visual function: Abbreviations: $\mathrm{BG}=$ Basal ganglia, $\mathrm{CB}=$ Cerebellum), $\mathrm{ION}=$ Inferior olivary nucleus, $\mathrm{LC}=$ Locus caeruleus, $\mathrm{MC}=$ Motor cortex, $\mathrm{PMC}=$ Premotor cortex, $\mathrm{PT}=$ Pontine nuclei, $\mathrm{SC}=$ Spinal cord, $\mathrm{SN}=$ Substantia nigra, $\mathrm{Th}=$ Thalamus

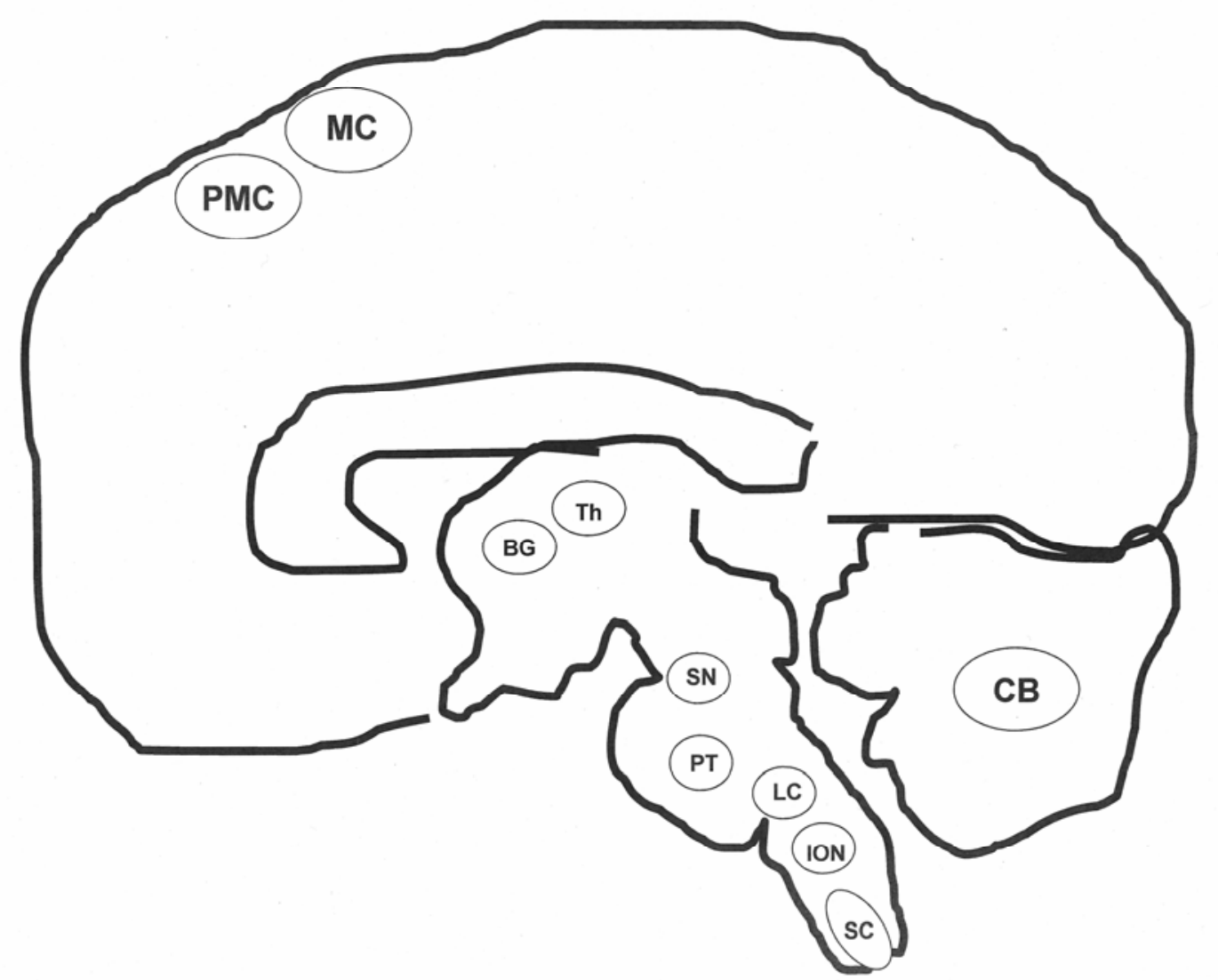

